Experimental Data

For

\title{
Base Mediated Reaction of Bestmann-Ohira Reagent with Nitroalkenes for the Regioselective Synthesis of Phosphonylpyrazoles
}

\author{
R. Muruganantham, ${ }^{a}$ Shaikh M. Mobin, ${ }^{\mathrm{b}}$ Irishi N. N. Namboothiri ${ }^{\mathrm{a}^{*}}$ \\ ${ }^{a}$ Department of Chemistry, Indian Institute of Technology, Bombay, Mumbai 400 076, INDIA, ${ }^{b}$ National \\ Single Crystal X-ray Diffraction Facility, Indian Institute of Technology, Bombay, Mumbai 400 076, INDIA
}

airishi@iitb.ac.in

Table of Contents

\begin{tabular}{|c|c|c|}
\hline & & Page \\
\hline 1 & Brief write-up on the optimization experiments (Table 1, main text) & 1 \\
\hline 2 & $\begin{array}{l}\text { Table S1. }{ }^{1} \mathrm{H} \text { and }{ }^{31} \mathrm{P} \text { NMR chemical shifts for the pyrazole tautomers } 5 \text { and } \\
\mathbf{5}^{\prime} \text { in } \mathrm{CDCl}_{3} \text { and DMSO- } \mathrm{d}_{6}\end{array}$ & 2 \\
\hline 3 & Experimental Section & $3-11$ \\
\hline 4 & Figure 1. Intermolecular H-bonding in solid state (X-ray) in $\mathbf{5 a}$ & 12 \\
\hline 5 & $\begin{array}{l}\text { Figure 2. Infinite network of ntermolecular H-bonding in solid state (X-ray) } \\
\text { in } \mathbf{5 a}\end{array}$ & 13 \\
\hline 6 & References & 14 \\
\hline
\end{tabular}

\section{Brief write-up on the optimization experiments (Table 1, main text)}

Treating nitroalkene 4a with BOR 2 in ethanol in the absence of any base provided no cycloadduct even under prolonged reflux conditions (entries 2 and 3). Since the yield in the $\mathrm{K}_{2} \mathrm{CO}_{3}$ mediated reaction was moderate $(7 \mathrm{~h}, 65 \%)$, we screened other bases such as $\mathrm{Et}_{3} \mathrm{~N}$, DABCO, DBU, NaOEt etc in ethanol as solvent (entries 4-7). Among these DBU and NaOEt provided pyrazole $5 \mathbf{a}$ in $63 \%(15 \mathrm{~h})$ and $77 \%(15 \mathrm{~min})$ yield, respectively (entries 6 and 7). Changing the base from $\mathrm{NaOEt}$ to $\mathrm{NaOMe}$ and solvent from ethanol to methanol led to substantial reduction in the yield due to trans-esterification of the phosphonate moiety (entry 8 ). When the DBU mediated reaction was carried out in aprotic solvents, either no reaction or complex mixture was encountered (entries 9-12). Low yield and prolonged reaction time were major problems when the NaOEt mediated reaction was conducted in an aprotic solvent (e.g. THF, entry 13). 
Table S1. ${ }^{1} \mathrm{H}$ and ${ }^{31} \mathrm{P}$ NMR chemical shifts (ppm) for the pyrazole $\mathrm{C}-\mathrm{H}$ in tautomers $\mathbf{5}$ and $\mathbf{5}$, in $\mathrm{CDCl}_{3}$ and DMSO-d $\mathrm{d}_{6}$.<smiles></smiles>

\begin{tabular}{|c|c|c|c|c|c|c|c|c|}
\hline \multirow[t]{2}{*}{ entry } & \multirow[t]{2}{*}{ pyrazole } & \multirow[t]{2}{*}{$\boldsymbol{R}$} & \multicolumn{3}{|c|}{$\begin{array}{c}{ }^{I} H N M R C(5) H \\
(298 K)\end{array}$} & \multicolumn{3}{|c|}{$\begin{array}{l}{ }^{31} P N M R \\
(298 \mathrm{~K})\end{array}$} \\
\hline & & & $\mathrm{CDCl}_{3}{ }^{\mathrm{a}}$ & $\begin{array}{l}D M S O \\
-d_{6}\end{array}$ & $\begin{array}{l}5^{\prime}: 5 \text { in } \\
D M S O- \\
d 6\end{array}$ & $\mathrm{CDCl}_{3}$ & $\begin{array}{l}D M S O \\
-d_{6}\end{array}$ & $\begin{array}{l}5^{\prime}: 5 \text { in } \\
D M S O \\
-d 6\end{array}$ \\
\hline 1 & $5 \mathbf{a}$ & $3,4-\left(\mathrm{OCH}_{2} \mathrm{O}\right) \mathrm{Ph}$ & $\begin{array}{l}7.88 \\
7.89\end{array}$ & $\begin{array}{l}7.83 \\
\mathbf{8 . 1 0}\end{array}$ & $1: 4.3$ & 7.30 & $\begin{array}{l}7.78 \\
\mathbf{1 2 . 0 7}\end{array}$ & $1: 3.1$ \\
\hline 2 & $5 \mathbf{b}$ & $(4-\mathrm{OMe}) \mathrm{Ph}$ & $\begin{array}{l}7.80 \\
7.81\end{array}$ & $8.04^{b}$ & $-b$ & 7.52 & $7.86^{\mathrm{b}}$ & $1: 2.5$ \\
\hline 3 & $5 c$ & $(4-\mathrm{Cl}) \mathrm{Ph}$ & $7.87^{b}$ & $\begin{array}{l}7.92 \\
\mathbf{8 . 2 3}\end{array}$ & $1: 3.1$ & 7.30 & $\begin{array}{l}7.29 \\
11.61\end{array}$ & $1: 4$ \\
\hline 4 & $5 d$ & $\left(4-\mathrm{NO}_{2}\right) \mathrm{Ph}$ & $8.14^{b}$ & $\begin{array}{l}8.00 \\
\mathbf{8 . 4 5}\end{array}$ & 1.3 .8 & 11.23 & $\begin{array}{l}6.70 \\
\mathbf{1 1 . 0 9}\end{array}$ & $1: 4.1$ \\
\hline 5 & $5 e$ & $\left(3-\mathrm{NO}_{2}\right) \mathrm{Ph}$ & $\begin{array}{l}8.10 \\
8.11\end{array}$ & $\begin{array}{l}8.51 \\
8.57\end{array}$ & $1: 3.5$ & 7.36 & $\begin{array}{l}6.84 \\
\mathbf{1 1 . 2 3}\end{array}$ & $1: 4.3$ \\
\hline 6 & $5 f$ & $\left(2-\mathrm{NO}_{2}\right) \mathrm{Ph}$ & $\mathrm{b}, \mathrm{c}$ & $8.04^{b}$ & $-b$ & $-{ }^{d}$ & $\begin{array}{l}5.87 \\
9.98\end{array}$ & $1: 3.3$ \\
\hline 7 & $5 g$ & $\mathrm{Ph}$ & $\begin{array}{l}8.02 \\
8.03\end{array}$ & $\begin{array}{l}7.89 \\
8.18\end{array}$ & $1: 2.8$ & 8.20 & $\begin{array}{l}7.60 \\
11.89\end{array}$ & $1: 2.9$ \\
\hline 8 & $5 \mathrm{~h}$ & 2-Furyl & $8.13^{b}$ & $\begin{array}{l}7.94 \\
\mathbf{8 . 2 3}\end{array}$ & $1: 3.1$ & 6.62 & $\begin{array}{l}6.52 \\
\mathbf{1 0 . 7 0}\end{array}$ & $1: 2.8$ \\
\hline 9 & $5 \mathbf{i}$ & 2-Thienyl & $\begin{array}{l}7.90 \\
7.91\end{array}$ & $\begin{array}{l}7.87 \\
8.19\end{array}$ & $1: 2.8$ & 5.56 & $\begin{array}{l}9.37 \\
\mathbf{1 3 . 4 8}\end{array}$ & $1: 2.7$ \\
\hline 10 & $5 \mathbf{j}$ & $\mathrm{NMe}_{2}$ & $\begin{array}{l}7.30 \\
7.31\end{array}$ & $7.30^{b}$ & $-b$ & 9.35 & $12.11^{\mathrm{b}}$ & $-b$ \\
\hline
\end{tabular}

${ }^{\mathrm{a}}$ The two tautomers were in $\sim 1: 1$ ratio in $\mathrm{CDCl}_{3} ;{ }^{\mathrm{b}}$ unresolved (broad) peak; ${ }^{\mathrm{c}}$ recorded in $\mathrm{MeOH}-\mathrm{d}_{4}{ }^{1} \mathrm{H} \delta 7.85 ;{ }^{\mathrm{d}}$ insoluble in $\mathrm{CDCl}_{3}$. Due to partial overlapping and/or broadening of peaks corresponding to major and minor tautomers in ${ }^{1} \mathrm{H} N \mathrm{NR}$, the ratios obtained from ${ }^{31} \mathrm{P}$ NMR appear to be more dependable. 


\section{Experimental Section}

\section{General}

The melting points are uncorrected. IR spectra were recorded on a Perkin Elmer Spectrum One FT spectrometer. NMR spectra $\left({ }^{1} \mathrm{H},{ }^{13} \mathrm{C}\right.$ and $\left.{ }^{31} \mathrm{P}\right)$ were recorded on an AMX-400 or VXR-300S spectrometer. TMS was the internal standard for ${ }^{1} \mathrm{H}$ and ${ }^{13} \mathrm{C}$ and phosphoric acid was the external standard for ${ }^{31} \mathrm{P}$. The coupling constants $(J$ values) are given in Hz. High resolution mass spectra were recorded at $60-70 \mathrm{eV}$ on a Waters Micromass Q-TOF spectrometer (ESI, Ar). Nitroalkenes 4a-i were prepared in the laboratory by the standard nitroaldol (Henry) reaction. ${ }^{1}$ Nitroalkene $\mathbf{4 j}$ was also prepared following literature method. ${ }^{2} \beta$ Nitronaphthalene 11 was purchased from Aldrich.

Suitable X-ray quality crystals of 5a were grown and X-ray diffraction studies were undertaken. X-ray crystallographic data were collected from single crystal samples of volume $0.25 \times 0.15 \times 0.13 \mathrm{~mm}^{3}$. Unit cell dimensions were obtained using 25 centered reflections in $\theta$ range 5.1600-10.4600 mounted on a Nonius MACH 3 diffractometer equipped with graphite monochromated Mo K $\alpha$ radiation $(0.71073 \AA)$. The intensity data were collected by $\omega-2 \theta$ scan mode, and corrected by Lorentz Polarization and absorption effects using Psi-Scan ( $\psi-$ scan). Three standard reflections monitored after every 200 reflections and 3 intensity control reflections monitored every hour showed no significant changes $(<3 \%)$. The structure was solved by direct methods shelxs97 and refined by full-matrix least squares against $\mathrm{F}^{2}$ using shelx197 software. Non-hydrogen atoms were refined with anisotropic thermal parameters. All hydrogen atoms were geometrically fixed and allowed to refine using a riding model.

\section{General procedure for the 1,3-DC reaction of nitroalkene 4,10,11 or 14 with BOR 2}

To a stirred solution of nitroalkene 4, 10, 11 or $14(1 \mathrm{mmol})$ and BOR 2 (264 mg, $1.2 \mathrm{mmol})$ in dry EtOH $(5 \mathrm{~mL})$ was added NaOEt $(1.5 \mathrm{mmol})$ at room temperature and the resulting mixture was stirred until the reaction was complete (monitored by TLC). The reaction mixture was diluted with water $(15 \mathrm{~mL})$, neutralized with $5 \% \mathrm{HCl}(10 \mathrm{~mL})$ and extracted with ethylacetate $(3 \times 15 \mathrm{~mL})$. The combined organic layers were then washed with brine $(2 \times 10$ $\mathrm{mL}$ ), dried over anhyd. $\mathrm{Na}_{2} \mathrm{SO}_{4}$ and then concentrated in vacuo. The crude residue was purified by column chromatography (silica gel, $n$-hexane/ethylacetate: $3 / 1$ ) to afford pure pyrazole $5,12,13,15$ or 16 .

\section{Diethyl 4-(benzo[d][1,3]dioxol-5-yl)-1H-pyrazol-3-yl-3-phosphonate (5a)}

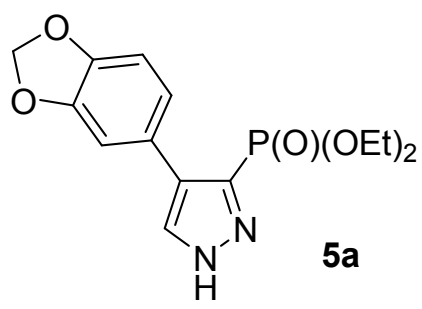


Colorless solid; Yield $77 \%$; mp $175{ }^{\circ} \mathrm{C}$; IR (KBr, cm $\left.{ }^{-1}\right) 3553$ (w), 3483 (w), 3410 (w), 3161 (s), $3118(\mathrm{~s}), 1484(\mathrm{~m}), 1400(\mathrm{~m}), 1220(\mathrm{~s}), 1023(\mathrm{~s}) ;{ }^{1} \mathrm{H} \mathrm{NMR}\left(\mathrm{CDCl}_{3}\right) \delta 1.23(\mathrm{t}, J=7.1 \mathrm{~Hz}$, $6 \mathrm{H}), 4.01-4.21(\mathrm{~m}, 4 \mathrm{H}), 5.99(\mathrm{~s}, 2 \mathrm{H}), 6.84(\mathrm{~d}, J=7.6 \mathrm{~Hz}, 1 \mathrm{H}), 7.06(\mathrm{~m}, 2 \mathrm{H}), 7.85 / 7.86(\mathrm{~s} / \mathrm{s}$, two tautomers in $\sim 1: 1$ ratio, $1 \mathrm{H}) ;{ }^{1} \mathrm{H}$ NMR $\left(\right.$ DMSO-d $\left._{6}\right) \delta 1.13(\mathrm{t}, J=7.0 \mathrm{~Hz}, 6 \mathrm{H}), 4.03(\mathrm{~m}$, 4H), 6.00 (s, 2H), $6.90(\mathrm{~d}, J=8.0 \mathrm{~Hz}, 1 \mathrm{H}), 7.11(\mathrm{~d}, J=8.0 \mathrm{~Hz}, 1 \mathrm{H}), 7.22(\mathrm{~s}, 1 \mathrm{H}), 7.83 / 8.10$ $(\mathrm{s} / \mathrm{s}$, minor/major in 1:4.3 ratio, $1 \mathrm{H}), 13.60(\mathrm{~s}, 1 \mathrm{H}) ;{ }^{13} \mathrm{C}$ NMR $\left(\mathrm{DMSO}^{-} \mathrm{d}_{6}\right) \delta 16.0\left(\mathrm{~d}, J_{\mathrm{C}-P}=6.1\right.$ $\mathrm{Hz}$ ), 62.2, 101.0, 108.2, 108.8, 121.7, 125.9, 128.8 (br), 136.5 (br), 138.8 (br), 146.3, 147.2; ${ }^{31} \mathrm{P}\left\{{ }^{1} \mathrm{H}\right\}$ NMR $\left(\mathrm{CDCl}_{3}\right) \delta 7.03 ;{ }^{31} \mathrm{P}\left\{{ }^{1} \mathrm{H}\right\}$ NMR $\left(\right.$ DMSO-d $\left._{6}\right) \delta$ 7.79/12.07 (minor/major in 1:3.1 ratio); MS (ESI) m/e (rel. intensity) $325\left(\mathrm{MH}^{+}, 100\right), 141$ (5); HRMS (ESI) calcd for $\mathrm{C}_{14} \mathrm{H}_{18} \mathrm{~N}_{2} \mathrm{O}_{5} \mathrm{P}\left(\mathrm{MH}^{+}\right)$325.0953, found 325.0967. See Ref. 25, main text, for selected X-ray data and CIF file complete data.

\section{Diethyl 4-(4-methoxyphenyl)-1H-pyrazol-3-yl-3-phosphonate (5b)}

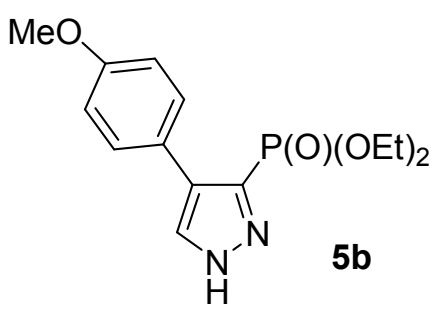

Colorless solid; Yield $66 \%$; mp 148-150 ${ }^{\circ} \mathrm{C}$; IR (KBr, $\left.\mathrm{cm}^{-1}\right) 3480$ (br m), 3137 (s), 3108 (s), 2985 (s), 2928 (s), 1228 (s), 1047 (s), 1022 (s); ${ }^{1} \mathrm{H} \mathrm{NMR}\left(\mathrm{CDCl}_{3}\right) \delta 1.21(\mathrm{t}, J=7.0 \mathrm{~Hz}, 6 \mathrm{H})$, $3.84(\mathrm{~s}, 3 \mathrm{H}), 3.84-4.17(\mathrm{~m}, 4 \mathrm{H}), 6.93(\mathrm{~d}, J=8.5 \mathrm{~Hz}, 2 \mathrm{H}), 7.52(\mathrm{~d}, J=8.5 \mathrm{~Hz}, 2 \mathrm{H}), 7.80 / 7.81$ $(\mathrm{s} / \mathrm{s}, 1 \mathrm{H}$, two tautomers in $1: 1 \mathrm{ratio}) ;{ }^{1} \mathrm{H}$ NMR (DMSO-d $\left.{ }_{6}\right) \delta 1.13(\mathrm{t}, J=6.9 \mathrm{~Hz}, 6 \mathrm{H}), 3.76(\mathrm{~s}$, $3 \mathrm{H}$ ), 3.99 (dq collapsed to quintet, $J=6.9 \mathrm{~Hz}, 4 \mathrm{H}), 6.94(\mathrm{~d}, J=8.5 \mathrm{~Hz}, 2 \mathrm{H}), 7.56(\mathrm{~d}, J=8.5$ $\mathrm{Hz}, 2 \mathrm{H}), 8.04$ (br s, 1H), 13.61 (br s, 1H); ${ }^{13} \mathrm{C}$ NMR (DMSO-d 6 ) $\delta 16.0(\mathrm{br}), 55.1,62.1$ (d, $J_{C-}$ $\left.{ }_{P}=6.1 \mathrm{~Hz}\right), 113.2$ (br), 114.0 (br), 124.3, 126.3 (d, $\left.J_{C-P}=22.8 \mathrm{~Hz}\right), 129.0$ (br), 129.9 (br), 158; ${ }^{31} \mathrm{P}\left\{{ }^{1} \mathrm{H}\right\}$ NMR $\left(\mathrm{CDCl}_{3}\right) \delta 7.52 ;{ }^{31} \mathrm{P}\left\{{ }^{1} \mathrm{H}\right\}$ NMR (DMSO-d $\left.{ }_{6}\right) \delta$ 7.87/12.12 (minor/major in $1: 2.5$ ratio); MS (ESI) $m / e$ (rel. intensity) $311\left(\mathrm{MH}^{+}, 100\right), 155$ (25), 124 (62); HRMS (ESI) calcd for $\mathrm{C}_{14} \mathrm{H}_{20} \mathrm{~N}_{2} \mathrm{O}_{4} \mathrm{P}\left(\mathrm{MH}^{+}\right)$311.1161, found 311.1162.

\section{Diethyl 4-(4-chlorophenyl)-1H-pyrazol-3-yl-3-phosphonate (5c)}

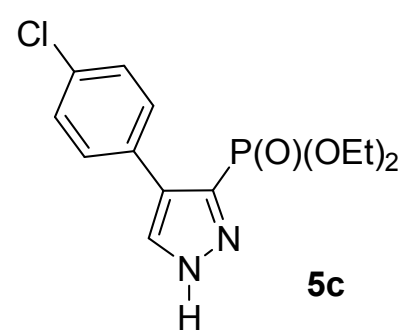

Colorless solid; Yield $61 \%$; mp $183{ }^{\circ} \mathrm{C}$; IR (KBr, cm $\left.{ }^{-1}\right) 3160$ (w), 3149 (w), 3123 (w), 2925 $(\mathrm{m}), 1635(\mathrm{~s}), 1483(\mathrm{~m}), 1226(\mathrm{~s}), 1024(\mathrm{~m}) ;{ }^{1} \mathrm{H}$ NMR $\left(\mathrm{CDCl}_{3}\right) \delta 1.21(\mathrm{t}, J=7.0 \mathrm{~Hz}, 6 \mathrm{H})$, 4.00-4.21 (m, 4H), $7.37(\mathrm{~d}, J=8.5 \mathrm{~Hz}, 2 \mathrm{H}), 7.53(\mathrm{~d}, J=8.5 \mathrm{~Hz}, 2 \mathrm{H}), 7.87(\mathrm{~s}, 1 \mathrm{H}) ;{ }^{1} \mathrm{H}$ NMR $\left(\right.$ DMSO-d $\left._{6}\right) \delta 1.14(\mathrm{t}, J=6.9 \mathrm{~Hz}, 6 \mathrm{H}), 4.00(\mathrm{dq}$ collapsed to quintet, $J=6.9 \mathrm{~Hz}, 4 \mathrm{H}), 7.44(\mathrm{~d}$, 
$J=8.4 \mathrm{~Hz}, 2 \mathrm{H}), 7.66(\mathrm{~d}, J=8.4 \mathrm{~Hz}, 2 \mathrm{H}), 7.92 / 8.23(\mathrm{~s} / \mathrm{s}$, minor/major in 1:3.1 ratio, $1 \mathrm{H})$, $13.74(\mathrm{~s}, 1 \mathrm{H}) ;{ }^{13} \mathrm{C} \mathrm{NMR}\left(\mathrm{CDCl}_{3}\right) \delta 16.1,62.3,125.1$ (br), 128.3, 130.0, 131.0, 131.7, 136.9 (br), 138.9 (br); ${ }^{31} \mathrm{P}\left\{{ }^{1} \mathrm{H}\right\} \mathrm{NMR}\left(\mathrm{CDCl}_{3}\right) \delta 7.31 ;{ }^{31} \mathrm{P}\left\{{ }^{1} \mathrm{H}\right\} \mathrm{NMR} \quad\left(\mathrm{DMSO}-\mathrm{d}_{6}\right) \delta$ 7.30/11.62 (minor/major in 1:4 ratio); MS (ESI) m/e (rel. intensity) $315\left(\mathrm{MH}^{+}, 100\right), 287$ (20), 259 (35); HRMS (ESI) calcd for $\mathrm{C}_{13} \mathrm{H}_{17} \mathrm{~N}_{2} \mathrm{O}_{3} \mathrm{ClP}\left(\mathrm{MH}^{+}\right)$315.0665, found 315.0658 .

\section{Diethyl 4-(4-nitrophenyl)-1H-pyrazol-3-yl-3-phosphonate (5d)}

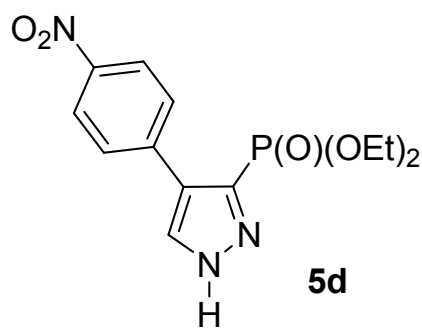

Colorless solid; Yield $64 \%$; mp $195{ }^{\circ} \mathrm{C}$; IR (KBr, cm ${ }^{-1}$ ) 3142 (br s), 3116 (br s), 2987 (m), 1605 (m), 1518 (s), 1340 (m), 1227 (s), 1047 (m), $1021(\mathrm{~s}) ;{ }^{1} \mathrm{H}$ NMR $\left(\mathrm{CDCl}_{3}\right) \delta 1.24(\mathrm{t}, J=$ $6.9 \mathrm{~Hz}, 6 \mathrm{H}), 4.10-4.22(\mathrm{~m}, 4 \mathrm{H}), 7.81(\mathrm{~d}, J=8.5 \mathrm{~Hz}, 2 \mathrm{H}), 8.13(\mathrm{~s}, 1 \mathrm{H}), 8.27(\mathrm{~d}, J=8.5 \mathrm{~Hz}$, 2H), 10.10 (br s, $1 \mathrm{H}) ;{ }^{1} \mathrm{H}$ NMR (DMSO-d 6 ) $\delta 1.16(\mathrm{t}, J=6.9 \mathrm{~Hz}, 6 \mathrm{H}), 4.05$ (dq collapsed to quintet, $J=6.9 \mathrm{~Hz}, 4 \mathrm{H}), 7.95(\mathrm{~d}, J=8.7 \mathrm{~Hz}, 2 \mathrm{H}), 8.26\left(\mathrm{~d}, J_{C-P}=8.7 \mathrm{~Hz}, 2 \mathrm{H}\right), 8.09 / 8.45(\mathrm{~s} / \mathrm{s}$, minor/major in 1:3.8 ratio, $1 \mathrm{H}), 13.93(\mathrm{~s}, 1 \mathrm{H}) ;{ }^{13} \mathrm{C}$ NMR $\left(\mathrm{DMSO}_{6}\right) \delta 16.0(\mathrm{~d}, J=6.1 \mathrm{~Hz})$, 62.5, 123.6, $124.0\left(\mathrm{~d}, J_{C-P}=23.6 \mathrm{~Hz}\right), 129.0,130.5,139.1,139.6,146.0 ;{ }^{31} \mathrm{P}\left\{{ }^{1} \mathrm{H}\right\} \mathrm{NMR}$ $\left(\mathrm{CDCl}_{3}\right) \delta 3.57 ;{ }^{31} \mathrm{P}\left\{{ }^{1} \mathrm{H}\right\} \mathrm{NMR}$ (DMSO-d 6 ) $\delta$ 6.70/11.09 (minor/major in 1:4.1 ratio); MS (ESI) $m / e$ (rel. intensity) $348\left(\mathrm{MNa}^{+}, 100\right)$, HRMS (ESI) calcd for $\mathrm{C}_{13} \mathrm{H}_{16} \mathrm{~N}_{3} \mathrm{O}_{5} \mathrm{PNa}\left(\mathrm{MNa}^{+}\right)$ 348.0725, found 348.0723.

\section{Diethyl 4-(3-nitrophenyl)-1H-pyrazol-3-yl-3-phosphonate (5e)}

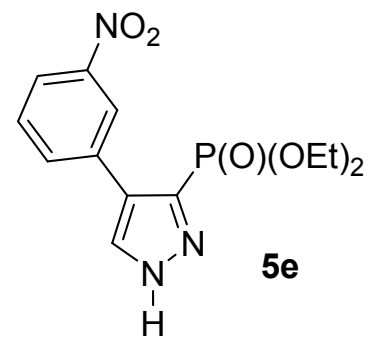

Colorless solid; Yield $61 \%$; mp $132{ }^{\circ} \mathrm{C}$; IR (KBr, cm ${ }^{-1}$ ) 3162 (br s), 3117 (br s), 3117 (s), $2983(\mathrm{~m}), 1546$ (m), 1522 (s), 1352 (m), 1229 (s), $1056(\mathrm{~m}), 1023(\mathrm{~s}) ;{ }^{1} \mathrm{H}$ NMR $\left(\mathrm{CDCl}_{3}\right) \delta$ $1.25(\mathrm{t}, J=6.9 \mathrm{~Hz}, 6 \mathrm{H}), 4.08-4.27(\mathrm{~m}, 4 \mathrm{H}), 7.59(\mathrm{t}, J=8.0 \mathrm{~Hz}, 1 \mathrm{H}), 7.94(\mathrm{dt}, J=8.0,0.7 \mathrm{~Hz}$, $1 \mathrm{H}), 8.10 / 8.11(\mathrm{~s} / \mathrm{s}, 1 \mathrm{H}$, two tautomers in $1: 1 \mathrm{ratio}), 8.20(\mathrm{ddd}, J=8.0,2.2,0.7 \mathrm{~Hz}, 1 \mathrm{H}), 8.55$ $(\mathrm{t}, J=2.2 \mathrm{~Hz}, 1 \mathrm{H}) ;{ }^{1} \mathrm{H}$ NMR $\left(\mathrm{DMSO}_{6}\right) \delta 1.74(\mathrm{t}, J=7.1 \mathrm{~Hz}, 6 \mathrm{H}), 4.04$ (dq collapsed to quintet, $4 \mathrm{H}), 7.67-7.75(\mathrm{~m}, 1 \mathrm{H}), 8.04-8.22(\mathrm{~m}, 2 \mathrm{H}), 8.44(\mathrm{~m}, 1 \mathrm{H}), 8.52 / 8.80$ (br s/br s, minor/ major in 1:3.6 ratio, $1 \mathrm{H}), 13.88 / 13.97$ (br s/br s, major/minor in $3.6: 1$ ratio, $1 \mathrm{H})$; ${ }^{13} \mathrm{C} \mathrm{NMR}$ $\left(\mathrm{CDCl}_{3}\right) \delta 16.1\left(\mathrm{~d}, J_{C-P}=6.1 \mathrm{~Hz}\right), 63.0(\mathrm{~d}, J=5.4 \mathrm{~Hz}), 122.1,123.6\left(\mathrm{~d}, J_{C-P}=3.1 \mathrm{~Hz}\right), 124.8$, 125.0, 129.3, 133.5, 133.8, 134.7, 148.2; ${ }^{31} \mathrm{P}\left\{{ }^{1} \mathrm{H}\right\} \mathrm{NMR}\left(\mathrm{CDCl}_{3}\right) \delta \quad 7.37 ;{ }^{31} \mathrm{P}\left\{{ }^{1} \mathrm{H}\right\} \mathrm{NMR}$ 
$\left(\right.$ DMSO-d $\left._{6}\right) \delta$ 6.84/11.24 (minor/major in 1:4.3 ratio); MS (ESI) $\mathrm{m} / \mathrm{e}$ (rel. intensity) 326.09 $\left(\mathrm{MH}^{+}, 100\right)$; HRMS (ESI) calcd for $\mathrm{C}_{13} \mathrm{H}_{17} \mathrm{~N}_{3} \mathrm{O}_{5} \mathrm{P}\left(\mathrm{MH}^{+}\right)$326.0906, found 326.0911.

\section{Diethyl 4-(2-nitrophenyl)-1H-pyrazol-3-yl-3-phosphonate (5f)}

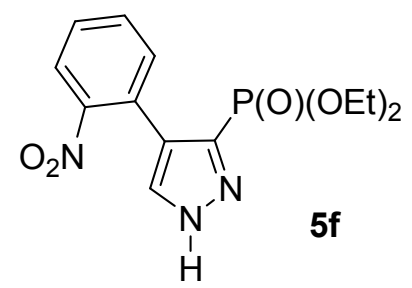

Colorless solid; Yield $67 \%$; mp 222-224 ${ }^{\circ} \mathrm{C}$; IR (KBr, cm $\left.{ }^{-1}\right) 3438$ (br m), 3154 (br s), 3123 (br s), 2982 (m), 2909 (m), 1522 (s), 1352 (m), 1227 (s), 1050 (m), 1021 (m); ${ }^{1} \mathrm{H}$ NMR $\left(\right.$ DMSO-d $\left._{6}\right) \delta 1.07(\mathrm{t}, J=7.1 \mathrm{~Hz}, 6 \mathrm{H}), 3.78-3.97(\mathrm{~m}, 4 \mathrm{H}), 7.47(\mathrm{dd}, \mathrm{J}=7.7,1.5 \mathrm{~Hz}, 1 \mathrm{H}), 7.58-$ $7.63(\mathrm{~m}, 1 \mathrm{H}), 7.69-7.74(\mathrm{~m}, 1 \mathrm{H}), 8.02(\mathrm{~m}, 1 \mathrm{H}), 8.04(\mathrm{br} \mathrm{s}, 1 \mathrm{H}), 13.79 / 13.96(\mathrm{br} \mathrm{s}$, major/minor in 3.3:1 ratio, $1 \mathrm{H}) ;{ }^{1} \mathrm{H}$ NMR $\left(\mathrm{CD}_{3} \mathrm{OD}\right) \delta 1.16(\mathrm{t}, J=6.9 \mathrm{~Hz}, 6 \mathrm{H}), 3.92-4.06(\mathrm{~m}, 4 \mathrm{H}), 7.48-$ $7.51(\mathrm{~m}, 1 \mathrm{H}), 7.56-7.62(\mathrm{~m}, 1 \mathrm{H}), 7.65-7.68(\mathrm{~m}, 1 \mathrm{H}), 7.85(\mathrm{br} \mathrm{s}, 1 \mathrm{H}), 8.01(\mathrm{dd}, J=8.1,1.1 \mathrm{~Hz}$, $1 \mathrm{H}) ;{ }^{13} \mathrm{C}$ NMR (DMSO-d $)_{6} \delta 16.7\left(\mathrm{~d}, J_{C-P}=6.1 \mathrm{~Hz}\right), 62.6,122.6\left(\mathrm{~d}, J_{C-P}=22.9 \mathrm{~Hz}\right), 124.9$, 127.9, 129.9, 130.3, 133.4, 134.4, 140.7, 150.0; ${ }^{31} \mathrm{P}\left\{{ }^{1} \mathrm{H}\right\} \mathrm{NMR}\left(\mathrm{DMSO}_{\mathrm{d}} \mathrm{d}_{6} \delta\right.$ 5.88/9.98 (minor/major in 1:3.3 ratio); MS (ESI) m/e (rel. intensity) 387 (]MNaK] $\left.]^{+}, 20\right), 371$ (22), 304 (15), 253 (28), 159 (10), 147 (38), 99 (62); HRMS (ESI) calcd for $\mathrm{C}_{13} \mathrm{H}_{16} \mathrm{~N}_{3} \mathrm{O}_{5} \mathrm{PNaK}$ $\left([\mathrm{MNaK}]^{+}\right) 387.0362$, found 387.0363 .

\section{Diethyl 4-phenyl-1H-pyrazol-3-yl-3-phosphonate (5g)}

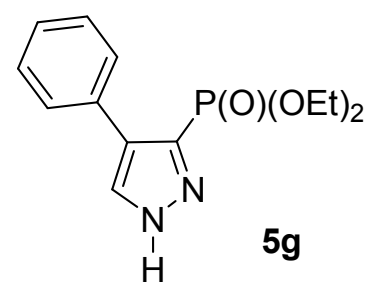

Colorless solid; Yield $62 \%$; mp $147{ }^{\circ} \mathrm{C}$; IR (KBr, cm $\left.{ }^{-1}\right) 3181$ (br m), 3115 (m), 1229 (s), $1021(\mathrm{~s}) ;{ }^{1} \mathrm{H}$ NMR $\left(\mathrm{CDCl}_{3}\right) \delta 1.17(\mathrm{t}, J=7.3 \mathrm{~Hz}, 6 \mathrm{H}), 3.98-4.20(\mathrm{~m}, 4 \mathrm{H}), 7.27-7.42(\mathrm{~m}, 3 \mathrm{H})$, 7.57-7.62 (m, 2H), 8.02/8.03 (s/s, two tautomers in $1: 1$ ratio); ${ }^{1} \mathrm{H}$ NMR $\left(\right.$ DMSO-d $\left.\mathrm{d}_{6}\right) \delta 1.13(\mathrm{t}$, $J=7.3 \mathrm{~Hz}, 6 \mathrm{H}), 3.99(\mathrm{~m}, 4 \mathrm{H}), 7.25-7.45(\mathrm{~m}, 3 \mathrm{H}), 7.57-7.68(\mathrm{~m}, 2 \mathrm{H}), 7.89 / 8.18(\mathrm{~s} / \mathrm{s}$, minor/major in 1:2.8 ratio, $1 \mathrm{H}), 13.67(\mathrm{~s}, 1 \mathrm{H}) ;{ }^{13} \mathrm{C} \mathrm{NMR}\left(\mathrm{CDCl}_{3}\right) \delta 15.9\left(\mathrm{~d}, J_{C-P}=6.9 \mathrm{~Hz}\right)$, $62.6\left(\mathrm{~d}, J_{C-P}=5.4 \mathrm{~Hz}\right), 127.2,127.3,127.5,128.2,128.6,131.7,134.4(\mathrm{br}) ;{ }^{31} \mathrm{P}\left\{{ }^{1} \mathrm{H}\right\} \mathrm{NMR}$ $\left(\mathrm{CDCl}_{3}\right) \delta 8.20 ;{ }^{31} \mathrm{P}\left\{{ }^{1} \mathrm{H}\right\}$ NMR (DMSO-d 6 ) $\delta$ 7.60/11.89 (minor/major in 1:2.9 ratio); MS (ESI) $m / e$ (rel. intensity) $303\left(\mathrm{MNa}^{+}, 100\right)$; HRMS (ESI) calcd for $\mathrm{C}_{13} \mathrm{H}_{17} \mathrm{~N}_{2} \mathrm{O}_{3} \mathrm{PNa}\left(\mathrm{MNa}^{+}\right)$ 303.0875, found 303.0872. 


\section{Diethyl 4-(2-furyl)-1H-pyrazol-3-yl-3-phosphonate (5h)}

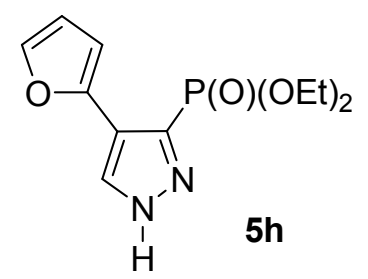

Colorless solid; Yield $55 \%$; mp $125^{\circ} \mathrm{C}$; IR (KBr, cm $\left.{ }^{-1}\right) 3138$ (br s), 3108 (s), 2988 (m), 2939 $(\mathrm{m}), 1230(\mathrm{~s}), 1200(\mathrm{~m}), 1045(\mathrm{~s}), 1020(\mathrm{~s}) ;{ }^{1} \mathrm{H}$ NMR $\left(\mathrm{CDCl}_{3}\right) \delta 1.29(\mathrm{t}, J=7.0 \mathrm{~Hz}, 6 \mathrm{H})$, 4.05-4.27 (m, 4H), 6.45 (dd, $J=3.3,1.8 \mathrm{~Hz}, 1 \mathrm{H}), 6.70$ (dd, $J=3.3,0.6 \mathrm{~Hz}, 1 \mathrm{H}), 7.42$ (dd, $J=$ $1.8,0.6 \mathrm{~Hz}, 1 \mathrm{H}), 8.13(\mathrm{~s}, 1 \mathrm{H}), 13.23(\mathrm{br} \mathrm{s}, 1 \mathrm{H}) ;{ }^{1} \mathrm{H}$ NMR $\left(\right.$ DMSO-d $\left._{6}\right) \delta 1.20(\mathrm{t}, J=7.1 \mathrm{~Hz}$, $6 \mathrm{H}$ ), 4.05 (dq collapsed to quintet, $J=7.1 \mathrm{~Hz}, 4 \mathrm{H}$ ), 6.50 (unresolved m, 1H), 6.87 (unresolved $\mathrm{m}, 1 \mathrm{H}), 7.64$ (unresolved $\mathrm{m}, 1 \mathrm{H}), 7.94 / 8.23(\mathrm{~s} / \mathrm{s}$, minor/major 1:3.1 ratio, $1 \mathrm{H}), 13.70(\mathrm{br} \mathrm{s}$, $1 \mathrm{H}) ;{ }^{13} \mathrm{C}$ NMR $\left(\mathrm{CDCl}_{3}\right) \delta 16.1(\mathrm{~d}, J=6.0 \mathrm{~Hz}), 62.8(\mathrm{~d}, J=4.5 \mathrm{~Hz}), 107.5,111.4,117.9\left(\mathrm{~d}, J_{C-}\right.$ $\left.{ }_{P}=20.0 \mathrm{~Hz}\right), 132.1,133.7,141.4,146.3 ;{ }^{31} \mathrm{P}\left\{{ }^{1} \mathrm{H}\right\} \mathrm{NMR}\left(\mathrm{CDCl}_{3}\right) \delta 6.62 ;{ }^{31} \mathrm{P}\left\{{ }^{1} \mathrm{H}\right\} \mathrm{NMR}$ $\left(\right.$ DMSO-d $\left._{6}\right) \delta 6.53 / 10.70$ (minor/major in 1:2.8 ratio); MS (ESI) $\mathrm{m} / e$ (rel. intensity) 293 ( $\left.\mathrm{MNa}^{+}, 100\right)$; $\mathrm{HRMS}$ (ESI) calcd for $\mathrm{C}_{11} \mathrm{H}_{15} \mathrm{~N}_{2} \mathrm{O}_{4} \mathrm{PNa}\left(\mathrm{MNa}^{+}\right)$293.0667, found 293.0674.

\section{Diethyl 4-(2-thienyl)-1 H-pyrazol-3-yl-3-phosphonate (5i)}

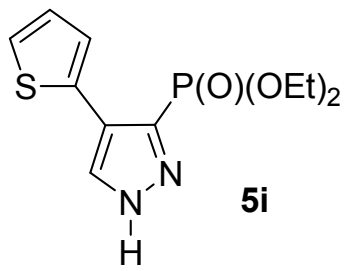

Colorless solid; Yield $49 \%$; mp $134{ }^{\circ} \mathrm{C}$; IR (KBr, cm $\left.{ }^{-1}\right) 3165$ (br s), 3116 (br s), 2985 (m), $2933(\mathrm{~m}), 1221(\mathrm{~s}), 1058(\mathrm{~s}), 1019(\mathrm{~s}) ;{ }^{1} \mathrm{H} \mathrm{NMR}\left(\mathrm{CDCl}_{3}\right) \delta 1.25(\mathrm{t}, J=7.1 \mathrm{~Hz}, 6 \mathrm{H}), 4.03-4.24$ (m, 4H), 7.06 (dd, $J=5.1,3.6 \mathrm{~Hz}, 1 \mathrm{H}), 7.28$ (dd, $J=5.1,1.2 \mathrm{~Hz}, 1 \mathrm{H}), 7.37$ (dd, $J=3.6,1.2$ $\mathrm{Hz}, 1 \mathrm{H}), 7.90 / 7.91(\mathrm{~s} / \mathrm{s}$, two tautomers in $1: 1 \mathrm{ratio}, 1 \mathrm{H}) ;{ }^{1} \mathrm{H}$ NMR $\left(\right.$ DMSO-d $\left._{6}\right) \delta 1.17(\mathrm{t}, J=$ $6.9 \mathrm{~Hz}, 6 \mathrm{H}), 4.01$ (dq collapsed to quintet, $J=6.9 \mathrm{~Hz}, 4 \mathrm{H}), 7.06-7.11(\mathrm{~m}, 1 \mathrm{H}), 7.38-7.52(\mathrm{~m}$, $2 \mathrm{H}), 7.87 / 8.19$ (br s/br s, minor/major in 1:2.8 ratio, $1 \mathrm{H}), 13.73 / 13.79$ ((br s/br s, major/minor in $2.5: 1$ ratio, $1 \mathrm{H}) ;{ }^{13} \mathrm{C}$ NMR $\left(\mathrm{CDCl}_{3}\right) \delta 16.1\left(\mathrm{~d}, J_{C-P}=6.9 \mathrm{~Hz}\right), 63.0\left(\mathrm{~d}, J_{C-P}=4.5 \mathrm{~Hz}\right), 120.5$ $\left(\mathrm{d}, J_{C-P}=20.6 \mathrm{~Hz}\right), 124.9,126.5,127.6,129.5,132.8,134.8(\mathrm{br}) ;{ }^{31} \mathrm{P}\left\{{ }^{1} \mathrm{H}\right\} \mathrm{NMR}\left(\mathrm{CDCl}_{3}\right) \delta$ 5.57; ${ }^{31} \mathrm{P}\left\{{ }^{1} \mathrm{H}\right\}$ NMR (DMSO-d 6 ) $\delta$ 9.31/13.48 (minor/major in 1:2.7 ratio); MS (ESI) m/e (rel. intensity) $287\left(\mathrm{MH}^{+}, 68\right), 259$ (20) HRMS (ESI) calcd for $\mathrm{C}_{11} \mathrm{H}_{16} \mathrm{~N}_{2} \mathrm{O}_{3} \mathrm{SP}\left(\mathrm{MH}^{+}\right)$287.0619, found 287.0612 . 


\section{Diethyl 4-(dimethylamino)-1H-pyrazol-3-yl-3-phosphonate (5j)}<smiles>CCOCOc1n[nH]cc1N(C)C</smiles>

Viscous liquid; Yield 64 \%; IR (KBr, cm $\left.{ }^{-1}\right) 3158$ (br s), 2981 (s), 2950 (s), 1544 (m), 1454 (w), $1391(\mathrm{w}), 1220(\mathrm{~s}), 1105(\mathrm{~m}), 1025(\mathrm{~s}) ;{ }^{1} \mathrm{H}$ NMR $\left(\mathrm{CDCl}_{3}\right) \delta 1.35(\mathrm{t}, J=6.8 \mathrm{~Hz}, 6 \mathrm{H}), 2.84$ $(\mathrm{s}, 6 \mathrm{H}), 4.08-4.24(\mathrm{~m}, 4 \mathrm{H}), 7.30 / 7.31(\mathrm{~s} / \mathrm{s}$, two tautomers in 1:1 ratio, $1 \mathrm{H}) ;{ }^{1} \mathrm{H}$ NMR (DMSO$\left.\mathrm{d}_{6}\right) \delta 1.13(\mathrm{t}, J=7.0 \mathrm{~Hz}, 6 \mathrm{H}), 2.57(\mathrm{~s}, 6 \mathrm{H}), 3.91$ (dq collapsed to quintet, $J=7.0 \mathrm{~Hz}, 4 \mathrm{H}$ ), 7.30 (br s, $1 \mathrm{H}), 12.98$ (br s, $1 \mathrm{H}) ;{ }^{13} \mathrm{C}$ NMR $\left(\mathrm{CDCl}_{3}\right) \delta 16.1(\mathrm{~d}, J=4.6 \mathrm{~Hz}), 44.9,62.4,122.5$ (br), 124.5 (br), 126.8 (br), 143.03 (d, $\left.J_{C-P}=19.1 \mathrm{~Hz}\right) ;{ }^{31} \mathrm{P}\left\{{ }^{1} \mathrm{H}\right\} \mathrm{NMR}\left(\mathrm{CDCl}_{3}\right) \delta$ 9.35; ${ }^{31} \mathrm{P}\left\{{ }^{1} \mathrm{H}\right\}$ NMR (DMSO-d $\left.\mathrm{d}_{6}\right) \delta 12.11$ (br); MS (ESI) m/e (rel. intensity) $270\left(\mathrm{MNa}^{+}, 5\right), 248$ (100), 220 (20); HRMS (ESI) calcd for $\mathrm{C}_{9} \mathrm{H}_{18} \mathrm{~N}_{3} \mathrm{O}_{3} \mathrm{PNa}\left(\mathrm{MNa}^{+}\right)$270.0983, found 270.0976.

\section{Diethyl 3,4-dihydro-4-(phenyl)chromeno[3,4-c]pyrazol-1-yl-1-phosphonate (12a)}

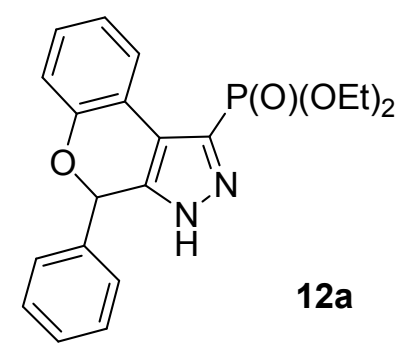

Colorless solid; Yield $57 \%$; mp $164{ }^{\circ} \mathrm{C}$; IR (KBr, cm $\left.{ }^{-1}\right) 3149$ (br s), 2925 (m), 1695 (m), $1650(\mathrm{~m}), 1558(\mathrm{~m}), 1539(\mathrm{~m}), 1515(\mathrm{~m}), 1474(\mathrm{~m}), 1247(\mathrm{~s}), 1014(\mathrm{~s}) ;{ }^{1} \mathrm{H}$ NMR $\left(\mathrm{CDCl}_{3}\right) \delta$ 1.29 (ABq, $J=6.9 \mathrm{~Hz}, 6 \mathrm{H}), 4.09-4.29(\mathrm{~m}, 4 \mathrm{H}), 6.45(\mathrm{~s}, 1 \mathrm{H}), 6.99-7.06(\mathrm{~m}, 2 \mathrm{H}), 7.16-7.20(\mathrm{~m}$, $1 \mathrm{H}), 7.28-7.34(\mathrm{~m}, 3 \mathrm{H}), 7.44-7.46(\mathrm{~m}, 2 \mathrm{H}) 7.82(\mathrm{dd}, J=7.6,1.5 \mathrm{~Hz}, 1 \mathrm{H}) ;{ }^{1} \mathrm{H}$ NMR (DMSO$\mathrm{d}_{6}$ ) $\delta 1.23$ (two partially overlapped ABq, $J=7.4 \mathrm{~Hz}, 6 \mathrm{H}$ ), 4.04-4.19 (m, 4H), 6.52/6.65 (br $\mathrm{s} / \mathrm{br} \mathrm{s}$, major/minor in $\sim 4.9: 1$ ratio, $1 \mathrm{H}), 6.99-7.04(\mathrm{~m}, 2 \mathrm{H}), 7.15-7.18(\mathrm{~m}, 1 \mathrm{H}), 7.35$ (unresolved m, 5H), 7.98/8.10 (br d, $J=6.9 \mathrm{~Hz}$, major/minor in $\sim 4.9: 1 \mathrm{ratio}, 1 \mathrm{H}$ ), 13.84 (br s, $1 \mathrm{H}) ;{ }^{13} \mathrm{C}$ NMR $\left(\mathrm{CDCl}_{3}\right) \delta 16.1(\mathrm{~d}, J=6.8 \mathrm{~Hz}), 63.4(\mathrm{~d}, J=3.8 \mathrm{~Hz}), 75.7,116.8\left(\mathrm{~d}, J_{C-P}=18.3\right.$ $\mathrm{Hz}), 117.6,117.8,122.1,123.6(\mathrm{br}), 125.6,127.2,128.4,128.4,128.9,139.1,148.3$ (d, $J_{C-P}=$ $15.2 \mathrm{~Hz}), 152.3 ;{ }^{31} \mathrm{P}\left\{{ }^{1} \mathrm{H}\right\}$ NMR $\left(\mathrm{CDCl}_{3}\right) \delta 5.19 ;{ }^{31} \mathrm{P}\left\{{ }^{1} \mathrm{H}\right\} \mathrm{NMR}\left(\mathrm{DMSO}-\mathrm{d}_{6}\right) \delta \quad 6.62,10.70$ (major/minor in 3.5:1 ratio); MS (ESI) m/e (rel. intensity) $385\left(\mathrm{MH}^{+}, 100\right) 357$ (28), 329 (20); HRMS (ESI) calcd for $\mathrm{C}_{20} \mathrm{H}_{22} \mathrm{~N}_{2} \mathrm{O}_{4} \mathrm{P}\left(\mathrm{MH}^{+}\right)$385.1317, found 385.1327. Due to partial overlapping and/or broadening of peaks corresponding to major and minor tautomers in ${ }^{1} \mathrm{H}$ NMR, the ratios obtained from ${ }^{31} \mathrm{P}$ NMR appear to be more dependable. 


\section{Diethyl 3,4-dihydro-4-(4-methoxyphenyl)chromeno[3,4-c]pyrazol-1-yl-1-phosphonate (12b)}

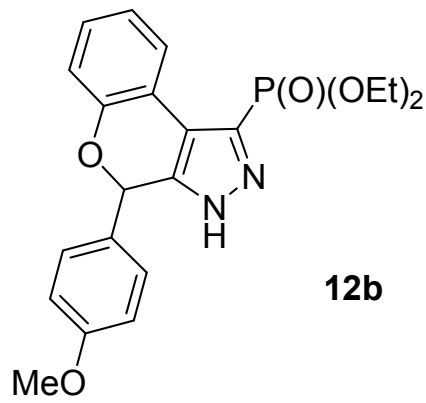

Highly viscous liquid; Yield 53 \%; IR $\left(\mathrm{KBr}, \mathrm{cm}^{-1}\right) 3435$ (br s), 2993 (s), 1638 (m), 1246 (s), 1218 (s), 1053 (s), 1029 (s); ${ }^{1} \mathrm{H}$ NMR $\left(\mathrm{CDCl}_{3}\right) \delta 1.31$ (two overlapped ABq, $J=7.0 \mathrm{~Hz}, 6 \mathrm{H}$ ), $3.77(\mathrm{~s}, 3 \mathrm{H}), 4.11-4.31(\mathrm{~m}, 4 \mathrm{H}), 6.37(\mathrm{~s}, 1 \mathrm{H}), 6.87(\mathrm{~d}, J=8.5 \mathrm{~Hz}, 2 \mathrm{H}), 6.91-7.03(\mathrm{~m}, 2 \mathrm{H})$, 7.16-7.21 (m, 1H), $7.36(\mathrm{~d}, J=8.5 \mathrm{~Hz}, 1 \mathrm{H}), 7.82-7.84(\mathrm{~m}, 1 \mathrm{H}) 12.11(\mathrm{br} \mathrm{s}, 1 \mathrm{H}) ;{ }^{1} \mathrm{H}$ NMR $\left(\right.$ DMSO-d $_{6}$ ) $\delta 1.24$ (two overlapped ABq, $J=7.0 \mathrm{~Hz}, 6 \mathrm{H}$ ), $3.72(\mathrm{~s}, 3 \mathrm{H}), 4.06-4.17(\mathrm{~m}, 4 \mathrm{H})$, $6.45 / 6.61$ (br s/br s, major/minor in $\sim 2.8: 1$ ratio, $1 \mathrm{H}), 6.90$ (d, $J=8.5 \mathrm{~Hz}, 2 \mathrm{H}), 6.95-7.03(\mathrm{~m}$, $2 \mathrm{H}$ ), 7.14-7.18 (m, 1H), 7.23 (d, $J=8.5 \mathrm{~Hz}, 2 \mathrm{H}), 7.98 / 8.12$ (br d, $J=7.5 \mathrm{~Hz}$, major/minor tautomers in $\sim 2.8: 1$ ratio, $1 \mathrm{H}), 13.77 / 13.84$ (br s/br s, minor/major tautomers in $\sim 1: 2.8$ ratio, $1 \mathrm{H}) ;{ }^{13} \mathrm{C}$ NMR $\left(\mathrm{CDCl}_{3}\right) \delta 16.05\left(\mathrm{~d}, J_{C-P}=6.8 \mathrm{~Hz}\right), 16.06\left(\mathrm{~d}, J_{C-P}=6.8 \mathrm{~Hz}\right), 55.1,63.2\left(\mathrm{~d}, J_{C-P}\right.$ $=4.6 \mathrm{~Hz}), 75.5,113.5,113.8,116.9\left(\mathrm{~d}, J_{C-P}=6.8 \mathrm{~Hz}\right), 117.6,117.7,122.0,125.5,128.7$, 128.8, 131.0, 148.6 (br), 152.3, 159.6; ${ }^{31} \mathrm{P}\left\{{ }^{1} \mathrm{H}\right\}$ NMR $\left(\mathrm{CDCl}_{3}\right) \delta 5.24 ;{ }^{31} \mathrm{P}\left\{{ }^{1} \mathrm{H}\right\}$ NMR (DMSO$\mathrm{d}_{6}$ ) $\delta$ 7.23/11.53 (major/minor in 2.7:1 ratio); MS (ESI) m/e (rel. intensity) 415 (100); HRMS (ESI) calcd for $\mathrm{C}_{21} \mathrm{H}_{24} \mathrm{~N}_{2} \mathrm{O}_{5} \mathrm{P}\left(\mathrm{MH}^{+}\right) 415.1423$, found 415.1415.

\section{Diethyl 3,4-dihydro-4-(2-furyl)chromeno[3,4-c]pyrazol-1-yl-1-phosphonate (12c)}

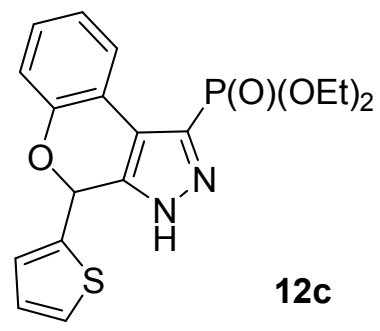

Colorless solid; Yield $55 \%$; mp 153-154 ${ }^{\circ} \mathrm{C}$; IR (KBr, cm $\left.{ }^{-1}\right) 3146$ (br s), 2987 (w), 2897 (w), $1475(\mathrm{~m}), 1434$ (w), 1247 (s), 1209 (s), 1013 (s); ${ }^{1} \mathrm{H}$ NMR $\left(\mathrm{CDCl}_{3}\right) \delta 1.28$ (ABq collapsed to $\mathrm{t}, J=7.0,3 \mathrm{H}), 1.33(\mathrm{ABq}$ collapsed to $\mathrm{t}, J=7.0 \mathrm{~Hz}, 3 \mathrm{H}), 4.07-4.33(\mathrm{~m}, 4 \mathrm{H}), 6.71(\mathrm{~s}, 1 \mathrm{H})$, $6.91(\mathrm{td}, J=3.7,1.5 \mathrm{~Hz}, 1 \mathrm{H}), 7.02(\mathrm{~m}, 3 \mathrm{H}), 7.18(\mathrm{~m}, 1 \mathrm{H}), 7.24(\mathrm{dd}, J=4.9,1.5 \mathrm{~Hz}, 1 \mathrm{H}), 7.82$ $(\mathrm{d}, J=7.5 \mathrm{~Hz}, 1 \mathrm{H}) ;{ }^{1} \mathrm{H}$ NMR $\left(\mathrm{DMSO}^{-} \mathrm{d}_{6}\right) \delta 1.23(\mathrm{ABq}$ collapsed to t, $J=7.0 \mathrm{~Hz}, 6 \mathrm{H}), 4.13$ (dq collapsed to quintet, $J=7.0 \mathrm{~Hz}, 4 \mathrm{H}), 6.82(\mathrm{~s}, 1 \mathrm{H}), 6.96-7.06(\mathrm{~m}, 4 \mathrm{H}), 7.17(\mathrm{t}, J=7.6 \mathrm{~Hz}$, $1 \mathrm{H}), 7.49 / 7.55(\mathrm{~d} / \mathrm{d}$, major/minor in $3.5: 1 \mathrm{ratio}, J=4.3 \mathrm{~Hz}, 1 \mathrm{H}), 7.98 / 8.12(\mathrm{~d} / \mathrm{d}$, major/minor 3.5:1 ratio, $J=7.6 \mathrm{~Hz}, 1 \mathrm{H}), 13.91(\mathrm{br} \mathrm{s}, 1 \mathrm{H}) ;{ }^{13} \mathrm{C} \mathrm{NMR}\left(\mathrm{CDCl}_{3}\right) \delta 16.3,63.6,71.6,116.9$ (d, 
$\left.J_{C-P}=17.6 \mathrm{~Hz}\right), 117.9,118.4,122.6,123.5,125.7,126.6,126.7,126.8,129.1,142.4,148.4$, $151.7 ;{ }^{31} \mathrm{P}\left\{{ }^{1} \mathrm{H}\right\}$ NMR $\left(\mathrm{CDCl}_{3}\right) \delta 5.12,{ }^{31} \mathrm{P}\left\{{ }^{1} \mathrm{H}\right\} \mathrm{NMR}\left(\mathrm{DMSO}-\mathrm{d}_{6}\right) \delta 6.96 / 11.03$ (major/minor tautomers in 3.9:1 ratio); MS (ESI) $m / e$ (rel intensity) $391\left(\left(\mathrm{MH}^{+}, 100\right)\right.$; HRMS (ESI) calcd for $\mathrm{C}_{18} \mathrm{H}_{20} \mathrm{~N}_{2} \mathrm{O}_{4} \mathrm{PS}\left(\mathrm{MH}^{+}\right)$391.0881, found 391.0885.

\section{Diethyl 3H-benzo[e]indazole-1-yl-1-phosphonate (13)}

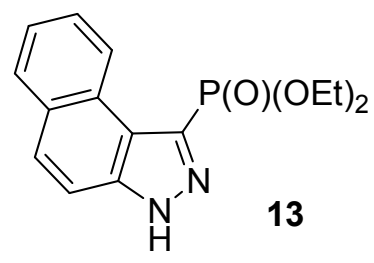

Colorless solid; Yield $38 \%$; mp $137^{\circ} \mathrm{C}$; IR (KBr, cm $\left.{ }^{-1}\right) 3164$ (br m), 2975 (m), 2922 (m), $1398(\mathrm{~m}), 1298(\mathrm{~m}), 1215(\mathrm{~s}), 1028(\mathrm{~s}) ;{ }^{1} \mathrm{H} \mathrm{NMR}\left(\mathrm{CDCl}_{3}\right) \delta 1.26(\mathrm{t}, J=7.0 \mathrm{~Hz}, 6 \mathrm{H}), 4.14-$ $4.30(\mathrm{~m}, 4 \mathrm{H}), 7.44-7.51(\mathrm{~m}, 3 \mathrm{H}), 7.85-7.87(\mathrm{~m}, 1 \mathrm{H}), 7.94(\mathrm{~d}, J=8.8 \mathrm{~Hz}, 1 \mathrm{H}), 8.62-8.65(\mathrm{~m}$, $1 \mathrm{H}) ;{ }^{1} \mathrm{H}$ NMR $\left(\mathrm{DMSO}_{-} \mathrm{d}_{6}\right) \delta 1.26(\mathrm{t}, J=6.9 \mathrm{~Hz}, 6 \mathrm{H}), 4.07-4.18(\mathrm{~m}, 4 \mathrm{H}), 7.63-7.75(\mathrm{~m}, 3 \mathrm{H})$, $7.88(\mathrm{~d}, J=8.8 \mathrm{~Hz}, 1 \mathrm{H}), 8.06$ (d, $J=7.9 \mathrm{~Hz}, 1 \mathrm{H}), 8.49$ (d, $J=7.9 \mathrm{~Hz}, 1 \mathrm{H}), 14.81$ (br s, $1 \mathrm{H}$ ); ${ }^{13} \mathrm{C}$ NMR $\left(\mathrm{CDCl}_{3}\right) \delta 16.3\left(\mathrm{~d}, J_{C-P}=6.9 \mathrm{~Hz}\right), 63.1\left(\mathrm{~d}, J_{C-P}=5.3 \mathrm{~Hz}\right), 118.8,120.8,122.7$, 122.9, 124.2, 126.7, 126.9, 128.6, 132.3, 133.0 (br), 135.4 (br), $139.2(\mathrm{br}) ;{ }^{31} \mathrm{P}\left\{{ }^{1} \mathrm{H}\right\} \mathrm{NMR}$ $\left(\mathrm{CDCl}_{3}\right) \delta 9.20 ;{ }^{31} \mathrm{P}\left\{{ }^{1} \mathrm{H}\right\}$ NMR (DMSO-d 6 ) $\delta$ 9.87; HRMS (ESI) calcd for $\mathrm{C}_{15} \mathrm{H}_{18} \mathrm{~N}_{2} \mathrm{O}_{3} \mathrm{P}(\mathrm{MH})$ 305.1055 , found 305.1053 .

\section{Diethyl nitro-4(phenyl)-1H-pyrazole-3-yl-3-phosphonate (15a)}

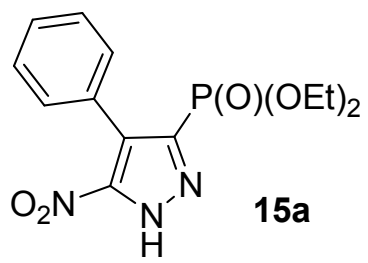

Colorless solid; Yield $47 \%$; mp $182{ }^{\circ} \mathrm{C}$; IR (KBr, cm $\left.{ }^{-1}\right) 3094$ (br s), 3059 (br s), 2987 (s), 2930 (s), 2894 (s), 1524 (s), 1399 (m), 1239 (s), 1215 (m), 1018 (s); ${ }^{1} \mathrm{H}$ NMR $\left(\mathrm{CDCl}_{3}\right) \delta 1.18$ (t, $J=7.0 \mathrm{~Hz}, 6 \mathrm{H}$ ), 3.98-4.15 (m, 4H), 7.38-7.47 (m, 5H), 14.19 (br s, $1 \mathrm{H}) ;{ }^{1} \mathrm{H}$ NMR (DMSO$\left.\mathrm{d}_{6}\right) \delta 1.05(\mathrm{t}, J=7.0 \mathrm{~Hz}, 6 \mathrm{H}), 3.85-3.98(\mathrm{~m}, 4 \mathrm{H}), 7.38-7.43(\mathrm{~m}, 5 \mathrm{H}), 14.9(\mathrm{br} \mathrm{s}, 1 \mathrm{H}) ;{ }^{13} \mathrm{C} \mathrm{NMR}$ $\left(\mathrm{CDCl}_{3}\right) \delta 15.8\left(\mathrm{~d}, J_{C-P}=6.9 \mathrm{~Hz}\right), 63.9\left(\mathrm{~d}, J_{C-P}=5.4 \mathrm{~Hz}\right), 123.4\left(\mathrm{~d}, J_{C-P}=17.6 \mathrm{~Hz}\right), 128.0$, $128.3,128.8,129.9,131.3,133.5,154.0\left(\mathrm{~d}, J_{C-P}=18.3 \mathrm{~Hz}\right) ;{ }^{31} \mathrm{P}\left\{{ }^{1} \mathrm{H}\right\} \mathrm{NMR}\left(\mathrm{CDCl}_{3}\right) \delta 0.40$, ${ }^{31} \mathrm{P}\left\{{ }^{1} \mathrm{H}\right\}$ NMR (DMSO-d $\left.\mathrm{d}_{6}\right) \delta 2.74$; MS (ESI) $m / e$ (rel. intensity) $348\left(\mathrm{MNa}^{+}, 10\right), 326\left(\mathrm{MH}^{+}\right.$, 100), 298 (20), 219 (12); HRMS (ESI) calcd for $\mathrm{C}_{13} \mathrm{H}_{17} \mathrm{~N}_{3} \mathrm{O}_{5} \mathrm{P}\left(\mathrm{MH}^{+}\right)$326.0906, found 326.0900 . 


\section{Diethyl 5-nitro-4(4-methoxyphenyl)-1H-pyrazole-3-yl-3-phosphonate (15b)}

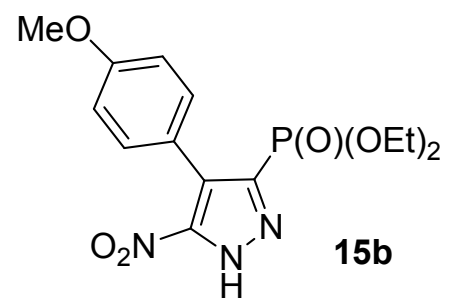

Light yellow solid; Yield $33 \%$; mp $180{ }^{\circ} \mathrm{C}$; IR (KBr, cm ${ }^{-1}$ ) 3093 (br m), 2926 (m), 1524 (s), $1395(\mathrm{~m}), 1243(\mathrm{~s}), 1021(\mathrm{~s}) ;{ }^{1} \mathrm{H} \mathrm{NMR}\left(\mathrm{CDCl}_{3}\right) \delta 1.18(\mathrm{t}, J=7.1 \mathrm{~Hz}, 6 \mathrm{H}), 3.87(\mathrm{~s}, 3 \mathrm{H}), 3.92-$ $4.16(\mathrm{~m}, 4 \mathrm{H}), 6.96(\mathrm{~d}, J=8.5 \mathrm{~Hz}, 2 \mathrm{H}), 7.31(\mathrm{~d}, J=8.5 \mathrm{~Hz}, 2 \mathrm{H}), 14.05$ (br s, $1 \mathrm{H}) ;{ }^{1} \mathrm{H}$ NMR $\left(\mathrm{DMSO}_{\mathrm{d}}\right) \delta 1.08(\mathrm{t}, J=7.0 \mathrm{~Hz}, 6 \mathrm{H}), 3.80(\mathrm{~s}, 3 \mathrm{H}), 3.86-3.99(\mathrm{~m}, 4 \mathrm{H}), 6.97(\mathrm{~d}, J=8.8 \mathrm{~Hz}$, $2 \mathrm{H}), 7.32(\mathrm{~d}, J=8.8 \mathrm{~Hz}, 2 \mathrm{H}) ;{ }^{13} \mathrm{C}$ NMR $\left(\mathrm{CDCl}_{3}\right) \delta 15.9\left(\mathrm{~d}, J_{C-P}=6.8 \mathrm{~Hz}\right), 55.4,63.90\left(\mathrm{~d}, J_{C-P}\right.$ $=5.3 \mathrm{~Hz}), 113.6,120.2,123.3\left(\mathrm{~d}, J_{C-P}=17.2 \mathrm{~Hz}\right), 131.3,133.4,154.2\left(\mathrm{~d}, J_{C-P}=18.3 \mathrm{~Hz}\right)$, 160.1; ${ }^{31} \mathrm{P}\left\{{ }^{1} \mathrm{H}\right\} \mathrm{NMR}\left(\mathrm{CDCl}_{3}\right) \delta 3.49 ;{ }^{31} \mathrm{P}\left\{{ }^{1} \mathrm{H}\right\} \mathrm{NMR}\left(\mathrm{DMSO}-\mathrm{d}_{6}\right) \delta$ 0.62; MS (ESI) m/e (rel. intensity) $356\left(\mathrm{MH}^{+}, 100\right)$; HRMS (ESI) calcd for $\mathrm{C}_{14} \mathrm{H}_{19} \mathrm{~N}_{3} \mathrm{O}_{6} \mathrm{P}\left(\mathrm{MH}^{+}\right)$356.1011, found 356.1009 .

\section{Diethyl-5-bromo-4(4-methoxyphenyl)-1H-pyrazole-3-yl-3-phosphonate (16b)}

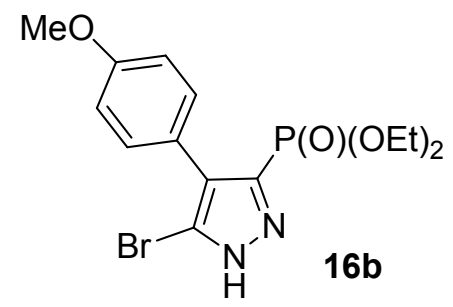

Colorless solid; Yield: $21 \%$; mp $151^{\circ} \mathrm{C}$; IR (KBr, cm $\left.{ }^{-1}\right) 3102$ (br s), 2953 (s), 2925 (s), 1230 (s), 1057 (s), $1029(\mathrm{~s}), 832(\mathrm{~m}), 562(\mathrm{~m}) ;{ }^{1} \mathrm{H}$ NMR $\left(\mathrm{CDCl}_{3}\right) \delta 1.17(\mathrm{t}, J=7.1 \mathrm{~Hz}, 6 \mathrm{H}), 3.85$ (s, $3 \mathrm{H}), 3.91-4.13(\mathrm{~m}, 4 \mathrm{H}), 6.96(\mathrm{~d}, J=8.8 \mathrm{~Hz}, 2 \mathrm{H}), 7.40$ (d, $J=8.8 \mathrm{~Hz}, 2 \mathrm{H}), 12.08$ (br s, $1 \mathrm{H})$; ${ }^{1} \mathrm{H}$ NMR $\left(\mathrm{DMSO}_{\mathrm{d}}\right) \delta 1.08(\mathrm{t}, J=7.0 \mathrm{~Hz}, 6 \mathrm{H}), 3.79(\mathrm{~s}, 3 \mathrm{H}), 3.84-4.00(\mathrm{~m}, 4 \mathrm{H}), 7.00(\mathrm{~d}, J=$ $8.8 \mathrm{~Hz}, 2 \mathrm{H}), 7.32(\mathrm{~d}, J=8.8 \mathrm{~Hz}, 2 \mathrm{H}) ;{ }^{13} \mathrm{C} \mathrm{NMR}\left(\mathrm{CDCl}_{3}\right) \delta 16.1\left(\mathrm{~d}, J_{C-P}=7.6 \mathrm{~Hz}\right), 55.4,63.5$ $\left(\mathrm{d}, J_{C-P}=5.3 \mathrm{~Hz}\right), 113.7,122.5,127.0\left(\mathrm{~d}, J_{C-P}=17.6\right), 128.2\left(\mathrm{~d}, J_{C-P}=19.8 \mathrm{~Hz}\right), 128.5,131.3$, 159.6; ${ }^{31} \mathrm{P}\left\{{ }^{1} \mathrm{H}\right\} \mathrm{NMR}\left(\mathrm{CDCl}_{3}\right) \delta 3.0 ;{ }^{31} \mathrm{P}\left\{{ }^{1} \mathrm{H}\right\} \mathrm{NMR}$ (DMSO-d $\left.\mathrm{d}_{6}\right) \delta 4.50$; MS (ESI) $\mathrm{m} / \mathrm{e}$ (rel. intensity) $391\left([\mathrm{MH}+2]^{+}, 100\right), 389\left(\mathrm{MH}^{+}, 100\right), 363$ (8), 361 (8); HRMS (ESI) calcd for $\mathrm{C}_{14} \mathrm{H}_{19} \mathrm{~N}_{2} \mathrm{O}_{4} \mathrm{PBr}^{79}\left(\mathrm{MH}^{+}\right)$389.0266, found 325.0248. 


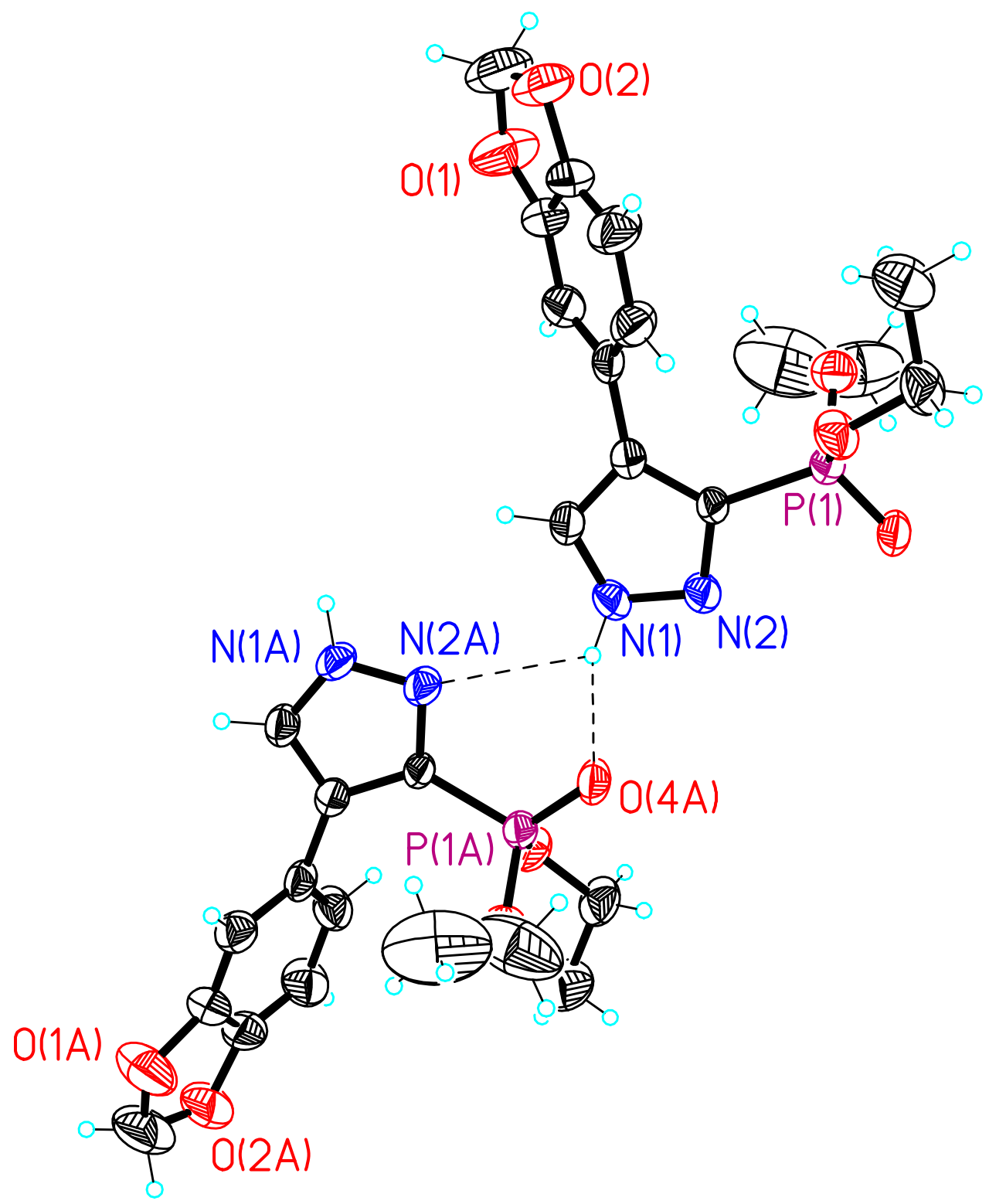

Figure 1. Intermolecular H-bonding in solid state (from X-ray) in 5a 


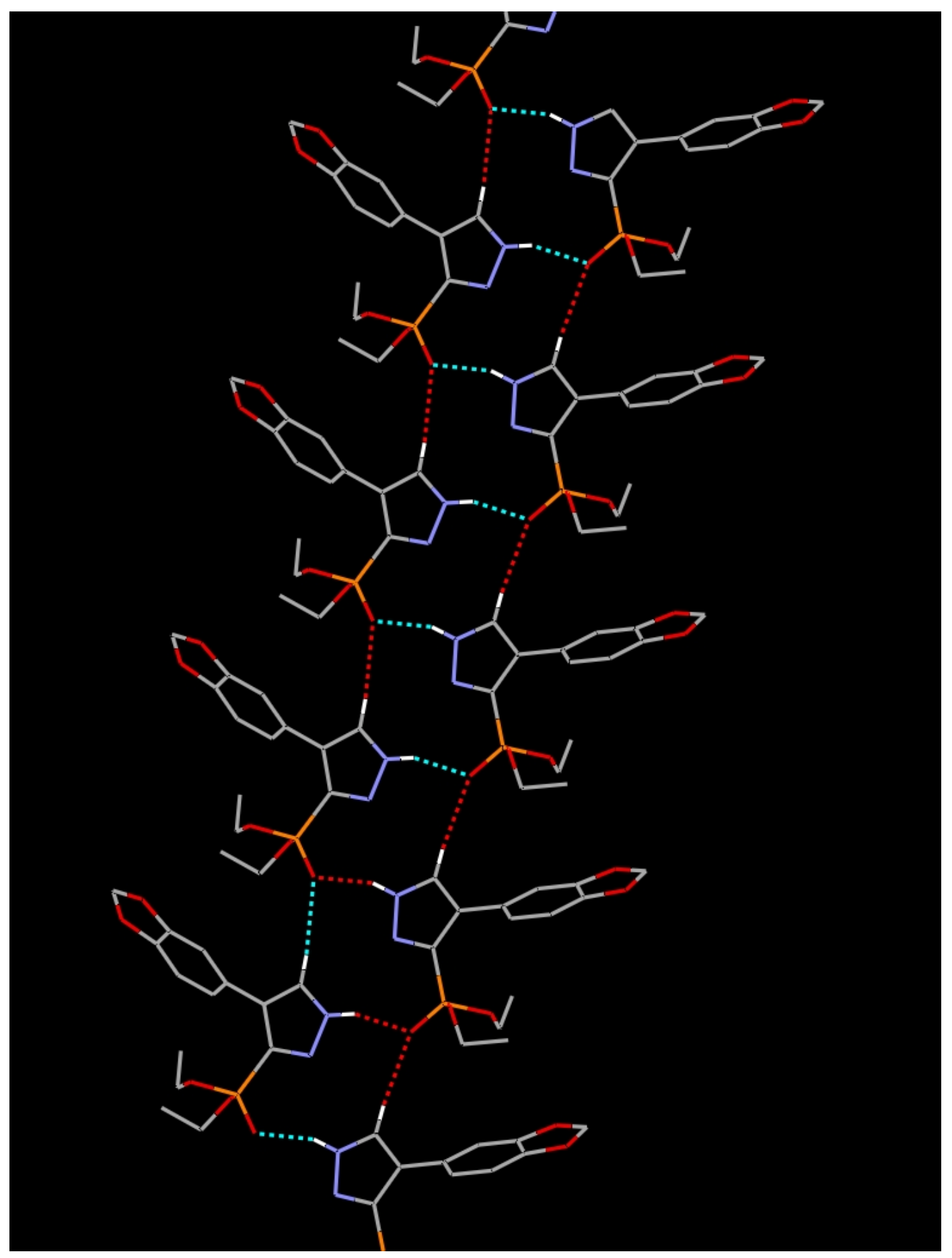

Figure 2. Infinite network of intermolecular H-bonding in solid state (from X-ray) in 5a 


\section{References}

(1) Vogel's Text Book of Practical Organic Chemistry, Addison Wesley Longman Ltd., Essex, England, $5^{\text {th }}$ Ed., 1989, p 1035.

(2) Nicolaou, K. C.; Snyder, S. A.; Huang, X.; Simonsen, K. B.; Koumbis, A. E.; Bigot, A.; J. Am. Chem. Soc. 2004, 126, 10162. 